\title{
Obligaciones estatales en el contexto de dietas no saludables: Allanando el camino dentro del Sistema Interamericano de Derechos Humanos ${ }^{1}$
}

\section{State Obligations in the Context of Unhealthy Diets: Paving the Way within the Inter-American Human Rights System}

\author{
Isabel Barbosa ${ }^{2}$ Belén Ríos $^{3}$ y Ariadna Tovar Ramírez ${ }^{4}$
}

Resumen: Las enfermedades crónicas no transmisibles (ENT) son la principal causa de morbilidad, mortalidad y discapacidad en las Américas. Las ENT son en gran medida prevenibles dada la naturaleza modificable de sus factores de riesgo, incluyendo el consumo elevado de productos procesados y ultra-procesados, asociado con las prácticas de la industria de productos comestibles y de bebidas. Este artículo explora los factores de riesgo de las ENT relacionados con la dieta como un asunto de derechos humanos que puede y debe ser

\footnotetext{
1 Traducción al español del original en inglés por Melissa Maria Agudelo Magalhaes. La traducción al español se realizó con el apoyo del Colectivo de Abogados José Alvear Restrepo (CAJAR). Las autoras agradecen la valiosa y profunda orientación proporcionada por Oscar A. Cabrera y Silvia Serrano Guzmán, así como el apoyo en la investigación que brindó Francesca Nardi.

2 Isabel Barbosa es Profesora Adjunta en Facultad de Derecho de la Universidad de Georgetown, Asociada en el O'Neill Institute for National and Global Health Law, y trabaja en el Global Center for Legal Innovation on Food Environments.

${ }^{3}$ Belén Ríos es Consultora en el O’Neill Institute for National and Global Health Law y trabaja en el Global Center for Legal Innovation on Food Environments.

${ }^{4}$ Ariadna Tovar Ramírez es Consultora en el O'Neill Institute for National and Global Health Law y trabaja en el Global Center for Legal Innovation on Food Environments.
} 
abordado en el ámbito del Sistema Interamericano de Derechos Humanos. Se argumenta que los Estados podrían ser responsables por incumplir la obligación de garantizar los derechos humanos; específicamente, al no actuar con la diligencia debida y regular la conducta de la industria de productos comestibles y bebidas. Además, se argumenta que los Estados también podrían resultar responsables por el incumplimiento de la obligación de respetar los derechos humanos, considerando su complicidad con la industria de productos comestibles y bebidas.

Palabras claves: Sistema interamericano de derechos humanos (SIDH); enfermedades crónicas no transmisibles (ENT); industria de productos comestibles y bebidas; debida diligencia; prevención; complicidad; captura corporativa.

Abstract: Noncommunicable diseases (NCDs) are the leading cause of morbidity, mortality, and disability in the Americas. NCDs are largely preventable because of the modifiable nature of their risk factors, including the elevated consumption of processed and ultraprocessed products that can be traced to the recurrent practices of the food and beverage industry. This article explores diet-related risk factors to NCDs as a human rights issue that can and should be addressed within the Inter-American Human Rights System (IAHRS). In particular, we argue that States can potentially be held responsible for their failure to comply with the obligation to guarantee human rights; specifically, by not acting with due diligence through the regulation of the food and beverage industry. Moreover, we argue that States can also potentially be held responsible for failing to comply with the obligation to respect human rights, considering its complicity with the food and beverage industry.

Keywords: Inter-American Human Rights System; noncommunicable diseases (NCDs); food and beverage industry; due diligence; prevention; complicity; corporate capture. 
Original en inglés: Recibido: 9.10.2020 Aceptado: 11.12.2020

Traducción: 26.03.2021

\section{Sumario}

\section{Introducción}

2. Factores de riesgo de las ENT relacionados con la dieta como un tema de derechos humanos

3. El papel de la industria de alimentos y bebidas en la epidemia de las ENT relacionadas con la dieta

4. Las obligaciones de garantizar y respetar los derechos humanos en el contexto de dietas no saludables dentro del Sistema Interamericano de Derechos Humanos

a. Obligación de garantizar los derechos humanos

b. Obligación de respetar los derechos humanos

\section{Conclusión}

\section{Introducción}

Nunca como en el marco de la Covid-19 se había debatido tanto la interacción entre el derecho, la salud y los derechos humanos en la región de las Américas. El impacto social y económico de esta pandemia no tiene precedentes, aunque todavía no es totalmente cuantificable (Organización Panamericana de la Salud 2020a). La crisis que atraviesa la salud en la actualidad ha impulsado a la comunidad jurídica a abordar una serie de cuestiones, como el acceso a atención médica, incluyendo la prevención y el tratamiento; el uso desproporcionado, discriminatorio o excesivo del derecho penal; y la necesidad de rendición de cuentas en todos los ámbitos (ONUSIDA 2020). Sin embargo, no se ha prestado suficiente atención a un tema crucial que la Covid-19 ha sacado a la luz: el impacto de los factores de riesgo de las enfermedades crónicas no transmisibles (ENT) relacionadas con la dieta, y de las empresas que los impulsan, en el disfrute de los derechos humanos. 
La Organización Panamericana de la Salud (OPS) define las ENT como un "grupo de enfermedades que no son causadas principalmente por una infección aguda, tienen como resultado consecuencias a largo plazo para la salud, y con frecuencia crean una necesidad de tratamiento y cuidados a largo plazo" (Organización Panamericana de la Salud 2020d). Las ENT afectan de manera desproporcionada a las personas de países de ingresos bajos y medianos, en los cuales se producen más de tres cuartas partes de las muertes mundiales por las ENT (32 millones). Son ejemplos de ENT los cánceres, las enfermedades cardiovasculares, las enfermedades respiratorias crónicas y la diabetes (Organización Mundial de la Salud 2018). En las Américas, las ENT son la principal causa de morbilidad, mortalidad y discapacidad, causando aproximadamente 5,5 millones de muertes por año, lo que representa el $80,7 \%$ de todas las muertes en la región. Además, del total de muertes por ENT, 38,9\% corresponden a muertes prematuras en personas menores de 70 años. En este sentido, las ENT representan una grave amenaza tanto para la salud pública como para el desarrollo social y económico (Organización Panamericana de la Salud 2019a).

Las ENT son en gran medida prevenibles, dado que existen diversos factores modificables que aumentan el riesgo de desarrollarlas (Organización Mundial de la Salud 2018). Uno de esos factores de riesgo son las dietas no saludables, estrechamente relacionadas con el sobrepeso, la obesidad, y las ENT relacionadas con la dieta (Organización Panamericana de la Salud 2020b). En este sentido, el Plan de acción mundial para la prevención y control de las enfermedades no transmisibles 2013-2020 estableció el objetivo de reducir los factores de riesgo para las ENT mediante la creación de entornos saludables, e incluyó una serie de recomendaciones para que los Estados fomentaran dietas saludables, algunas de las cuales no implicaban costos, o implicaban costos muy bajos, para los Estados (OMS 2013). En última instancia, como dice Gostin, "la tragedia moral radica en el hecho de que sí es posible prevenir, a un 
costo razonable, gran parte de este sufrimiento y de las muertes prematuras" (Gostin 2014).

Las dietas no saludables están asociadas con el exceso de ingesta de azúcares, grasas y sodio, lo cual, a su vez, se debe en gran medida a la amplia disponibilidad, asequibilidad y promoción de productos procesados y ultraprocesados (Organización Panamericana de la Salud 2020b). En este sentido, los retos que enfrenta la salud en este escenario se deben en parte al elevado consumo de alimentos procesados y ultraprocesados, que se puede atribuir a prácticas específicas y recurrentes de la industria de alimentos y bebidas. Estas empresas son ampliamente reconocidas como impulsoras clave de la epidemia de ENT relacionadas con la dieta en todo el mundo. Los productos alimenticios con poco valor nutricional se suelen fabricar para que sean baratos, sabrosos y fáciles de conservar durante largos períodos de tiempo, por lo cual son altamente rentables para la industria de alimentos y bebidas (Fredeunberg 2014). Estas empresas contribuyen a que haya entornos alimentarios poco saludables, y se benefician activamente de ellos.

La pandemia de la Covid-19 es un momento oportuno para provocar el debate en torno a los factores de riesgo de las ENT relacionados con la dieta. El vínculo entre la Covid-19 y las ENT ha sido bien documentado (UN Interagency Task Force on NCDs 2020), con evidencia que indica, por ejemplo, que la alta prevalencia de personas con obesidad exacerba la amenaza para su salud (Popkin et al. 2020). Entre tanto, también se han documentado las acciones de las empresas que están detrás de los productos no saludables, incluida la industria de alimentos y bebidas; acciones que van desde configurar los entornos en donde se adoptan políticas públicas, hasta desarrollar tácticas de mercadeo que aprovechan las condiciones creadas por la pandemia (NCD Alliance y Spectrum Consortium 2020). Este es un poderoso ejemplo de la compleja interacción entre las enfermedades infecciosas y las no transmisibles, la cual, aunque se conoce desde hace mucho tiempo, no se aborda de manera efectiva (Gostin 2014). 
Aunque últimamente se han hecho avances a nivel nacional para abordar los factores de riesgo de las ENT relacionados con la dieta, como la adopción e implementación de etiquetados frontales de advertencia en algunos países de las Américas (Organización Panamericana de la Salud 2020c), todavía queda mucho por hacer a nivel regional. Mediante un informe temático, la Comisión Interamericana de Derechos Humanos (Comisión Interamericana o $\mathrm{CIDH})$ ha comenzado a abordar en los últimos años el tema de las ENT, incluidos los factores de riesgo relacionados con la dieta (Comisión Interamericana de Derechos Humanos 2019a), en tanto que la Corte Interamericana de Derechos Humanos (Corte Interamericana) aún no ha analizado un solo caso ni ha emitido una opinión consultiva específica sobre este tema, como se analizará más adelante.

El presente artículo busca ayudar a llenar este vacío, analizando las obligaciones de garantía y respeto de los derechos humanos que tiene el Estado en relación con la industria de alimentos y bebidas dentro del Sistema Interamericano de Derechos Humanos (SIDH), basándose en los conceptos de debida diligencia y complicidad. Con este fin i) abordaremos la comprensión actual de los factores de riesgo de las ENT relacionados con la dieta como un problema de derechos humanos, tanto a nivel internacional como regional; ii) dilucidaremos el papel de la industria de alimentos y bebidas en la epidemia de las ENT relacionadas con la dieta; y (iii) analizaremos cómo la diligencia debida y la complicidad, tal como han evolucionado dentro del SIDH, se desarrollan en relación con las obligaciones del Estado de garantizar y respetar los derechos humanos en el contexto de las dietas poco saludables. Finalmente, concluiremos que los factores de riesgo de las ENT relacionados con la dieta son un problema de derechos humanos, que puede y debe ser abordado dentro del SIDH. 


\section{Factores de riesgo de las ENT relacionados con la dieta como un tema de derechos humanos}

Como lo analizaremos enseguida, los factores de riesgo de las ENT relacionados con la dieta son una cuestión de derechos humanos. Este es un tema complejo, que afecta a una diversidad de derechos humanos y sus obligaciones conexas. No obstante, no vamos a extender nuestro análisis a todos esos derechos humanos y obligaciones. En su lugar, nuestro objetivo en este punto es simplemente establecer que los factores de riesgo de las ENT relacionados con la dieta ya han sido reconocidos como un tema de derechos humanos tanto a nivel internacional como regional.

Los factores de riesgo de las ENT se han analizado a nivel internacional en múltiples ocasiones desde una perspectiva de derechos humanos, incluyendo la adopción de un tratado centrado en el control del tabaco (CMCT 2005), así como en declaraciones del Comité de Derechos Económicos, Sociales y Culturales como parte de los informes periódicos de país, sobre la salud y el consumo de tabaco en Argentina, en 2018, y sobre la salud y las ENT en Mauricio, en 2019. (Comité de Derechos Económicos, Sociales y Culturales 2018, 51-52; 2019, 51-52). En cuanto a los factores de riesgo de las ENT relacionados con la dieta, los ex Relatores Especiales de las Naciones Unidas sobre el derecho que le asiste a toda persona a disfrutar el más alto nivel posible de salud física y mental (Anand Grover 2014; Oficina del Alto Comisionado de las Naciones Unidas para los Derechos Humanos 2020), y los ex Relatores Especiales de las Naciones Unidas sobre el derecho al derecho a la alimentación (Jean Ziegler 2001; Hilal Elver 2016), han analizado el tema explícitamente desde una perspectiva de derechos humanos.

Desde el punto de vista del derecho a la salud, Grover publicó en 2014 un informe titulado Los alimentos poco saludables, las enfermedades no transmisibles y el derecho a la salud, en el que no solo enmarcó el tema de las dietas no saludables en relación con los 
derechos a la salud y a una alimentación adecuada, sino que también expuso a la industria de alimentos y bebidas por gastar miles de millones de dólares en "la promoción y comercialización continua y generalizada de alimentos poco saludables.” (Anand Grover 2014). A su vez, Pūras emitió hace pocos meses un pronunciamiento en el que se examinaba la adopción del etiquetado frontal de advertencia como una medida regulatoria muy necesaria para hacer frente a los factores de riesgo de las ENT relacionados con la dieta, respaldado por el Relator Especial de las Naciones Unidas sobre el derecho a la alimentación y el Grupo de Trabajo sobre la cuestión de los derechos humanos y las empresas transnacionales y otras empresas. Es importante señalar que Puras criticó en forma amplia la influencia indebida de la industria de alimentos y bebidas en la toma de decisiones gubernamentales. (Oficina del Alto Comisionado de las Naciones Unidas para los Derechos Humanos 2020).

Desde la perspectiva del derecho a una alimentación adecuada, Ziegler publicó en 2001 un informe en el que se refirió a la necesidad de una reforma legislativa, incluido el etiquetado, para proteger a los consumidores de alimentos considerados peligrosos (Jean Ziegler 2001). Además, en 2016, Elver publicó un informe sobre nutrición que abordaba la creciente amenaza de las ENT relacionadas con las dietas poco saludables, así como los efectos nocivos de la falta de regulación sobre las estrategias de mercadeo de productos alimenticios (Hilal Elver 2016). En particular, señaló que "las estrategias de mercadeo son particularmente perjudiciales cuando se dirigen a mercados sin explotar en países en desarrollo, un efecto indirecto de la 'saturación' de los mercados en los países desarrollados,” y agregó que "el efecto de introducir la comida rápida en la dieta de las poblaciones más pobres es especialmente peligroso cuando hay falta de conocimiento o educación, y cuando las personas son vulnerables a las prácticas manipuladoras del mercadeo." (Hilal Elver 2016, 34).

A nivel regional, en 2016, por primera vez el SIDH consideró directamente un factor de riesgo para las ENT, reconociendo que 
efectivamente se trataba de un asunto de derechos humanos que caía bajo su mandato. En esa ocasión, la Comisión Interamericana concedió al Instituto O’Neill para el Derecho de Salud Nacional y Global de la Facultad de Derecho de la Universidad de Georgetown, en colaboración con la Fundación Interamericana del Corazón Argentina y Action on Smoking and Health, una audiencia temática sobre el Derecho a la Salud y la Adicción al Tabaco en las Américas, que exploró la intersección entre el control del tabaco y los derechos humanos (Cabrera y Constantin 2020).

Sin embargo, no fue hasta 2019, con la publicación de un informe temático sobre Empresas y Derechos Humanos: Estándares Interamericanos (Comisión Interamericana de Derechos Humanos 2019a), de la Relatora Especial de la CIDH sobre Derechos Económicos, Sociales, Culturales y Ambientales, que la Comisión Interamericana abordó los factores de riesgo de las ENT relacionados con la dieta. Al tratar de la indebida influencia corporativa en la formulación de políticas, utilizó específicamente como ejemplo a la industria de alimentos y bebidas, refiriéndose a su presión en contra de la regulación sobre la comercialización de alimentos no saludables dirigida a los niños y las niñas, la adopción de etiquetas de advertencia en los alimentos ultraprocesados y la imposición de gravámenes a las bebidas azucaradas. En este sentido, la Comisión Interamericana señaló que tales dinámicas de poder tienen un mayor impacto en los grupos poblacionales que se encuentran en una situación vulnerable, comprometiendo los derechos a la salud, la alimentación, el agua y el medio ambiente (Comisión Interamericana de Derechos Humanos 2019a, 265).

Además, en el contexto de los derechos de los niños, niñas y adolescentes, la Comisión Interamericana destacó las preocupantes tasas de sobrepeso y obesidad en la región. Específicamente, indicó que este escenario puede derivarse de las acciones de las empresas, mediante el empleo de estrategias para aumentar las ventas, al mismo tiempo que obstaculizan la implementación de regulaciones conformes con los estándares de derechos humanos. Ejemplos de 
tales estrategias son el cabildeo y/o la presión ejercida sobre tomadores y tomadoras de decisiones, las amenazas de interponer demandas contra el Estado y la financiación de estudios que carecen de objetividad y apoyan sus propios intereses. En este sentido, la Comisión Interamericana recordó que los Estados deben tomar y hacer cumplir medidas para prevenir, abordar y sancionar de manera efectiva el impacto negativo de las actividades comerciales sobre los derechos de los niños, niñas y adolescentes. Además, las empresas deben ajustar sus procesos de toma de decisiones y sus operaciones tomando en cuenta el impacto que sus actividades comerciales tienen en los derechos de los niños, niñas y adolescentes (Comisión Interamericana de Derechos Humanos 2019a, 360-361).

Es importante destacar que, en el mencionado informe temático, la Comisión Interamericana también estableció la debida diligencia como un punto de referencia esencial en materia de empresas y derechos humanos, enfatizando de manera vehemente que no solo se refiere a las conductas requeridas de los Estados, sino también a las conductas que los Estados deben, a su vez, exigir a las empresas. Esto consistiría en un proceso de gestión continua, informado por las circunstancias particulares de la empresa, el sector en el que opera y el contexto en que desarrolla sus actividades. En este sentido, la debida diligencia radica en establecer sistemas y procesos eficaces para identificar, prevenir, mitigar y rendir cuentas de los daños que los Estados y las empresas causan, a los cuales contribuyen, o a los que de cualquier otro modo están vinculadas (Comisión Interamericana de Derechos Humanos 2019a, 37-38).

Con base en lo anterior, está claro que los factores de riesgo de las ENT relacionados con la dieta son ampliamente aceptados como un asunto de derechos humanos, tanto a nivel internacional como regional. Ahora dirigiremos nuestra atención a las empresas que impulsan dichos factores de riesgo. 


\section{El papel de la industria de alimentos y bebidas en la epidemia de las ENT relacionadas con la dieta}

El aumento de las ENT relacionadas con la dieta en la región no ocurrió por casualidad. Fue alimentado por las acciones de la industria de alimentos y bebidas, aprovechando la vía libre que recibieron de los Estados, como exploraremos a continuación.

El impacto de las empresas en la salud pública es ampliamente reconocido, tanto que llevó la academia a acuñar el término "determinantes comerciales de la salud", que es relevante en el contexto de dietas poco saludables, a pesar de aplicarse a una gama más amplia de empresas. Este concepto emergente se refiere a "estrategias y enfoques utilizados por el sector privado para promover productos ... que son perjudiciales para la salud" (Kickbusch et al. 2016, e895). Se acusa a las empresas de causar una "epidemia industrial" en la que las propias empresas son "vectores de enfermedad" (Mialon 2020, 4). Este marco ofrece una importante perspectiva alternativa a la noción generalizada de que las ENT son principalmente autoinfligidas; como han señalado Lee y Crosbie, que las personas simplemente deben darse cuenta de lo poco saludables que son sus hábitos (Lee y Crosbie 2020). Al hacerlo, arroja luz sobre el papel de las empresas, entre ellas la industria de alimentos y bebidas.

Inicialmente, es importante tener en cuenta que los alimentos y bebidas ultraprocesados se consideran generalmente poco saludables (Organización Panamericana de la Salud 2019b). La OPS los definió como "formulaciones industriales fabricadas principal o totalmente a partir de sustancias derivadas de los componentes de los alimentos, además de los aditivos utilizados para imitar e intensificar las cualidades sensoriales de los alimentos no procesados o mínimamente procesados" (Organización Panamericana de la Salud 2019b, 6). Estos productos son nutricionalmente desequilibrados, lo 
que significa que "[t]ienen un elevado contenido en azúcares libres, grasa total, grasas saturadas y sodio, y un bajo contenido en proteína, fibra alimentaria, minerales y vitaminas" (Organización Panamericana de la Salud 2019b, 2).

De manera particular, Fredeunberg señaló que estos "alimentos hiperplacenteros al paladar”, como los denominó, generalmente aumentan los beneficios de las empresas, en parte porque son altos en componentes baratos como grasas y azúcar, y en parte debido a los frecuentes subsidios a la producción de maíz, azúcar y soya (Fredeunberg 2014). Centrándose en la industria de las bebidas gaseosas, Nestle también se refirió a la rentabilidad de estas empresas, señalando la ironía de que reciben extraordinarios rendimientos de las bebidas azucaradas, mientras que fabricarlas les cuesta prácticamente nada, ya que su ingrediente principal, el agua, viene a bajo costo a expensas del Estado. Destacó que, en muchas estaciones de servicio, supermercados y cadenas de comida rápida, las bebidas gaseosas cuestan, de hecho, menos que las botellas de agua (Nestle, 2015).

La Covid-19 ha puesto de relieve las tácticas recurrentes utilizadas por la industria de alimentos y bebidas para aumentar sus ganancias a expensas de la salud pública. Durante la pandemia, estas empresas presentaron ofertas de colaboración a las autoridades, participaron en prácticas estratégicamente etiquetadas como "responsabilidad social empresarial" (RSE) o como filantropía, y utilizaron estrategias de mercadeo para aprovechar la pandemia en todo el mundo (NCD Alliance 2020). También intentaron capitalizar la pandemia truncando la implementación de medidas de salud pública, como el etiquetado frontal de advertencia en productos comestibles y bebidas en México (Grupo REFORMA 2020). Estas tácticas, aunque indignantes en medio de una pandemia empeorada debido a las altas tasas de sobrepeso, obesidad y ENT relacionadas con la dieta, están lejos de ser nuevas. Muchos actores de la sociedad civil, el mundo académico y los organismos internacionales las han venido documentando durante años. 
Por un lado, la industria de alimentos y bebidas emplea formas de comercialización y publicidad agresivas de productos poco saludables, influyendo en el comportamiento de las y los consumidores en el contexto de entornos poco saludables (Tangcharoensathien et al. 2019). Primero, la industria de alimentos y bebidas diseña "alimentos hiperagradables al gusto", que brindan a las y los consumidores mayores recompensas fisiológicas y psicológicas que los alimentos tradicionales, mezclando o colocando capas de grasa, sal y azúcar, además de incluir varios aditivos. Después, aumenta la exposición de las personas a tales productos a través de elaboradas estrategias de mercadeo (Fredeunberg 2014), que ejercen un profundo impacto en los patrones de compra y alimentación de las comunidades (Organización Panamericana de la Salud 2019b). En resumen, las empresas, incluidas las de alimentos y bebidas, han aprovechado este poder para hacer que sus productos parezcan más atractivos, aceptables y deseables para las y los consumidores (Kickbusch et al. 2016). Estas estrategias de mercadeo son particularmente efectivas en niños y adolescentes (Organización Panamericana de la Salud 2019b).

Por otro lado, la industria de alimentos y bebidas ha minado durante mucho tiempo las regulaciones acordes a los estándares de protección de los derechos humanos, dedicando una cantidad significativa de recursos para evitar la adopción e implementación de medidas que amenacen sus ganancias (Roache et al., 2018). Las tácticas incluyen cooptar legisladores y reguladores, financiar organizaciones que parecen tener una base comunitaria y a científicos y científicas que son amigables con sus intereses, así como litigar contra las medidas adoptadas por el Estado; todo para detener o debilitar regulaciones que probablemente sean efectivas para contener la alimentación no saludable (Gostin 2016). Una vez más, la obstaculización del etiquetado frontal de advertencia es un ejemplo poderoso que la sociedad civil ha documentado recientemente. A lo largo de los procesos de legislación, regulación e implementación en múltiples países, la industria de alimentos y bebidas ha utilizado 
diversas estrategias destinadas a obstruir esta medida regulatoria (Colectivo de Abogados José Alvear Restrepo y El Poder del Consumidor 2020).

$\mathrm{Al}$ oponerse ferozmente a las regulaciones concordantes con los estándares de protección de los derechos humanos, la industria de alimentos y bebidas ha quedado relativamente libre para formular, vender y comercializar productos no saludables, argumentando que puede regular su propio comportamiento para evitar daños (Gostin 2016). Sin embargo, no existe evidencia alguna de la efectividad de la autorregulación (Moodie et al. 2013). Por el contrario, hay investigaciones sustantivas que apuntan a la ineficacia de tales medidas (Organización Panamericana de la Salud 2011), que son "insuficientes en alcance y cobertura, utilizan criterios de nutrición débiles, y carecen de una aplicación y de sanciones suficientemente fuertes para asegurar su cumplimiento" (Global Food Research Program 2020, 9). En este sentido, Gostin advirtió que la autorregulación suele derivar en reglas que son altamente permisivas y, además, disuaden al Estado de actuar más enérgicamente (Gostin 2016).

Esta dinámica de oposición a las normas acordes con los derechos ha sido reconocida recientemente en la declaración antes mencionada de Puras, el ex Relator Especial de las Naciones Unidas sobre el derecho a la salud. Al hablar sobre el etiquetado frontal de advertencia, fue puntual al describir las prácticas de la industria de alimentos y bebidas:

Sin embargo, la industria de alimentos y bebidas sigue oponiéndose de manera firme y holgada a las regulaciones de etiquetado frontal de advertencia. Esto incluye encubrir los efectos nocivos de los productos alimenticios con cantidades excesivas de nutrientes críticos mediante múltiples tácticas, como el patrocinio de investigaciones para minimizar los vínculos con problemas de salud (...). En los casos en que los Estados han adoptado efectivamente reglamentos de etiquetado frontal de advertencia para promover la salud 
pública, algunas empresas han recurrido a litigios o han amenazado con hacerlo. También han recurrido a otras campañas y tácticas para retrasar y/o bloquear la aplicación de estas medidas reglamentarias, revocarlas o disminuir su efecto (Oficina del Alto Comisionado de las Naciones Unidas para los Derechos Humanos 2020).

Vale la pena señalar que Puras no solo describió las tácticas antes mencionadas, sino que reconoció expresamente que la desinformación y la presión ejercidas por la industria de alimentos y bebidas interfieren con los esfuerzos de los Estados para adoptar leyes, reglamentos y políticas de salud pública. Más importante aún, dijo que este comportamiento era un intento de "interferir o influir directamente en los procesos de toma de decisiones gubernamentales," calificándolo directamente como influencia indebida de las empresas en estos procesos de toma de decisiones. Por último, indicó que esta situación debería ser abordada por los Estados para garantizar normativas respetuosas de los derechos, que impidan la generación de daños para la salud de las personas derivados del consumo de alimentos y bebidas no saludables (Oficina del Alto Comisionado de las Naciones Unidas para los Derechos Humanos 2020).

La agresiva comercialización y publicidad por parte de la industria de alimentos y bebidas, junto con su obstaculización a las regulaciones estatales respetuosas de los derechos, deja en claro que la epidemia de las ENT relacionadas con la dieta no es aleatoria. Más bien, es el producto de la acción deliberada de la industria de alimentos y bebidas, cuya ocurrencia se ha permitido por la falta de adopción de medidas eficaces por parte de los Estados. 


\section{Las obligaciones de garantizar y respetar los derechos humanos en el contexto de dietas no saludables dentro del Sistema Interamericano de Derechos Humanos}

Las prácticas descritas anteriormente no dejan dudas sobre el papel de la industria de alimentos y bebidas en la epidemia de enfermedades no transmisibles relacionadas con la dieta y, por lo tanto, sobre el disfrute de los derechos humanos, especialmente los derechos a la salud y a una alimentación adecuada, como mínimo. Cabe destacar que la Corte Interamericana ya ha decidido que dichos derechos están protegidos de forma autónoma en virtud del artículo 26 de la Convención Americana sobre Derechos Humanos (Convención Americana) (Corte Interamericana de Derechos Humanos 2018a; 2018b; 2020a).

A pesar del avance incipiente en el abordaje directo de los factores de riesgo para las ENT, incluidos los relacionados con la dieta, la Comisión y la Corte Interamericanas han consolidado dos conceptos que resultan relevantes en este escenario: el primero es la diligencia debida, en relación con la obligación del Estado de garantizar los derechos humanos; y el segundo es la complicidad, que se manifiesta en forma de tolerancia, aquiescencia y colaboración. Ambos conceptos ayudan a definir el contenido y alcance de las obligaciones del Estado que se derivan del artículo 1.1 de la Convención Americana en relación con los derechos mencionados en el contexto de las dietas no saludables.

\section{a. Obligación de garantizar los derechos humanos}

La idea de la diligencia debida ha estado presente desde hace mucho tiempo en el derecho internacional, lo que significa que los Estados tienen el deber de respetar el derecho internacional y verificar que los agentes estatales y no estatales dentro de su jurisdicción hagan lo mismo (Cantú 2017). En las Américas, la Corte 
Interamericana estableció el deber de diligencia hace décadas, en Velázquez Rodríguez contra Honduras, explicando que:

en principio, es imputable al Estado toda violación a los derechos reconocidos por la Convención cumplida por un acto del poder público o de personas que actúan prevalidas de los poderes que ostentan por su carácter oficial. No obstante, no se agotan allí las situaciones en las cuales un Estado está obligado a prevenir, investigar y sancionar las violaciones a los derechos humanos, ni los supuestos en que su responsabilidad puede verse comprometida por efecto de una lesión a esos derechos. En efecto, un hecho ilícito violatorio de los derechos humanos que inicialmente no resulte imputable directamente a un Estado, por ejemplo, por ser obra de un particular o por no haberse identificado al autor de la trasgresión, puede acarrear la responsabilidad internacional del Estado, no por ese hecho en sí mismo, sino por falta de la debida diligencia para prevenir la violación o para tratarla en los términos requeridos por la Convención (Corte Interamericana de Derechos Humanos, 1988).

Hasta el día de hoy, este precedente ha guiado la jurisprudencia regional sobre la debida diligencia, consolidando su entendimiento como el deber de tomar todas las medidas necesarias para lograr la protección de los derechos humanos, incluyendo la investigación, sanción y reparación de los daños causados por actores privados (Cantú 2017).

No obstante, la interpretación de la debida diligencia se ha ampliado a lo largo de los años para abordar diferentes conjuntos de circunstancias; por ejemplo, centrándose en la prevención mediante enfoques específicos y generales. Por lo tanto, en esta forma preventiva de debida diligencia, la Corte Interamericana ha establecido y seguido criterios que se centran, principalmente, en la noción de riesgo a nivel individual o grupal (Corte Interamericana de Derechos Humanos 2009). En otras ocasiones, ha establecido deberes de regular, supervisar y monitorear a nivel poblacional. Ambos tipos 
de debida diligencia preventiva están relacionados, dado que una situación de riesgo generalizado ayuda a definir el carácter previsible y evitable de un riesgo particular (Abramovich 2010, 179).

El primer tipo, el enfoque específico de la debida diligencia preventiva, puede ser ejemplificado por González y otras (Campo Algodonero) Vs. México, un caso sobre las desapariciones forzadas de mujeres, en el cual la Corte Interamericana estableció los siguientes criterios en cuanto al riesgo: (i) debe haber una situación de riesgo real e inmediato; (ii) esta situación debe amenazar a una persona o grupo específico; (iii) el Estado debe saber o debería haber sabido de la existencia del riesgo; y (iv) el Estado podría haber prevenido o evitado razonablemente la materialización del riesgo (Cabrera 2020). En todos los casos, "debe atenderse a las circunstancias particulares del caso y a la concreción de dichas obligaciones de garantía” (Corte Interamericana de Derechos Humanos 2009, 280).

El segundo tipo, el enfoque general de la debida diligencia preventiva, se puede encontrar en una serie de decisiones en el contexto del derecho a la salud. En Ximenes Lopes Vs. Brasil, un caso sobre la muerte de una persona internada en una clínica psiquiátrica privada que funcionaba como parte del sistema de salud pública, la Corte Interamericana estableció que "los Estados tienen el deber de regular y fiscalizar toda la asistencia de salud prestada a las personas bajo su jurisdicción, como deber especial de protección a la vida y a la integridad personal, independientemente de si la entidad que presta tales servicios es de carácter público o privado" (Corte Interamericana de Derechos Humanos 2006, 89). Otros casos relacionados con la salud siguieron el mismo razonamiento (Corte Interamericana de Derechos Humanos 2013; 2015; 2018; 2019). Cabe destacar que en Gonzales Lluy y otros Vs. Ecuador, un caso sobre contagio del VIH a través de un banco de sangre cuyo manejo había sido delegado a la Cruz Roja, la Corte Interamericana destacó la gravedad de la afectación a la salud involucrada, así como los altos riesgos que la víctima podría haber enfrentado en diferentes momentos de su vida, entendiendo la obligación que el Estado tiene de garantizar los 
derechos humanos como una debida diligencia, de alguna manera, reforzada (Corte Interamericana de Derechos Humanos 2015).

La Corte Interamericana también ha aplicado el deber de regular, supervisar y monitorear en contextos distintos de la atención en salud que involucran a actores privados. En el Caso Trabajadores de la Hacienda Brasil Verde Vs. Brasil, sobre la esclavitud contemporánea, la Corte Interamericana analizó cómo la debida diligencia se manifiesta en el deber de inspección de actores privados (Corte Interamericana de Derechos Humanos 2016). En el Caso de las Comunidades Indígenas de la Asociación Lhaka Honhat (Nuestra Tierra) Vs. Argentina, sobre una disputa de tierras, la Corte Interamericana se refirió al deber de regular, supervisar y vigilar en relación con el derecho a una alimentación adecuada y otros derechos (Corte Interamericana de Derechos Humanos 2020a). Finalmente, en el Caso de los empleados de la fábrica de fuegos artificiales de Santo Antônio de Jesus Vs. Brasil, sobre una explosión en una fábrica de fuegos artificiales que mató a varias personas, la Corte Interamericana se refirió al deber de regular, supervisar y monitorear en el contexto de actividades peligrosas, explorando nuevamente las implicaciones de las actividades de alto riesgo en el disfrute de los derechos humanos (Corte Interamericana de Derechos Humanos 2020b).

El desarrollo de la debida diligencia dentro del SIDH se incorpora en gran medida en Empresas y Derechos Humanos: Estándares Interamericanos, que ocasionalmente establece paralelismos con los Principios Rectores de las Naciones Unidas sobre Empresas y los Derechos Humanos (Principios Rectores de las Naciones Unidas). Sobre este tema, es importante tener en cuenta que los Principios Rectores de las Naciones Unidas estudian la debida diligencia como parte de la responsabilidad de respetar los derechos humanos que recae en las empresas. Se describe como "un proceso continuo de gestión que una empresa prudente y razonable debe llevar a cabo, a la luz de sus circunstancias (como el sector en el que opera, el contexto en que realiza su actividad, su tamaño y otros factores) para hacer frente a su responsabilidad de respetar los derechos humanos" 
(Oficina del Alto Comisionado de las Naciones Unidas, 2012, 7). En particular, el principio rector 17 establece que la debida diligencia debe cubrir los impactos negativos sobre los derechos humanos que las empresas causan, contribuyen o a los que están directamente vinculadas, con el objetivo de identificar, prevenir, mitigar y rendir cuentas de dichos impactos. Además, este proceso debe variar tomando en cuenta consideraciones sobre la propia empresa y la naturaleza y el contexto de sus operaciones (Oficina del Alto Comisionado de las Naciones Unidas, 2012).

Esta definición se asemeja en muchos aspectos a la utilizada posteriormente por la Comisión Interamericana en el informe temático Empresas y Derechos Humanos: Estándares Interamericanos, como se describe en los apartados anteriores. Al explorar el papel de la debida diligencia en el contexto de las empresas y los derechos humanos, lo interpretó como un proceso continuo; fue más allá de la causalidad para incluir la contribución o la vinculación con el daño; estableció el objetivo de identificar, prevenir, mitigar y rendir cuentas por el daño; e indicó que este proceso debe ser informado por las circunstancias particulares de la empresa, el sector en el que opera y el contexto que rodea a sus actividades. Sin embargo, hay una diferencia crucial: en Empresas y Derechos Humanos: Estándares Interamericanos, el enfoque de la debida diligencia no radica en la responsabilidad corporativa, sino en las obligaciones del Estado, en la medida en que se requiere la debida diligencia de los Estados, que, a su vez, deben exigir determinadas conductas de las empresas (Comisión Interamericana de Derechos Humanos 2019a, 37-38).

En este sentido, la Comisión Interamericana se basó en la línea jurisprudencial sólida y arraigada, discutida anteriormente, centrándose en el hecho de que los Estados están obligados principalmente a garantizar los derechos humanos y, por lo tanto, pueden ser considerados responsables de no actuar con la debida diligencia en relación con actores no estatales. Al hacerlo, la Comisión Interamericana explicó cómo la obligación de garantizar los derechos 
humanos se manifiesta en varios deberes en el contexto de las empresas y los derechos humanos (Comisión Interamericana de Derechos Humanos 2019a). Aquí, nos centramos en el deber de regular debido a su particular importancia a la luz de las prácticas de la industria de alimentos y bebidas descritas anteriormente; específicamente, la obstaculización recurrente de la adopción y/o implementación de regulaciones concordantes con los estándares de protección de los derechos humanos.

El deber de regular incluye no solo la supresión de actos normativos o prácticas que atenten contra los derechos humanos, sino también la adopción de legislación interna y políticas pertinentes que defiendan los derechos humanos en relación con la actividad corporativa en cuestión (Comisión Interamericana de Derechos Humanos 2019a, 104). En el contexto de las empresas y los derechos humanos, esto significa adaptar el marco legal tanto en materia sustantiva como procesal, incluido el derecho administrativo, civil y penal, así como su aplicabilidad extraterritorial (Comisión Interamericana de Derechos Humanos 2019a, 111). En última instancia, la regulación de las empresas consiste en un enfoque estructural de la debida diligencia, facilitando y reforzando el correspondiente deber de prevención (Comisión Interamericana de Derechos Humanos 2019a, 89-90).

Además, la Comisión Interamericana destacó el desequilibrio entre las empresas y las personas afectadas por sus actividades, reflexionando sobre la influencia de las primeras, en detrimento de las segundas, en los procesos institucionales a través de los que se adoptan las regulaciones. Por lo tanto, los Estados deben garantizar espacios de transparencia y participación efectiva cuando se trata de marcos jurídicos; espacios en los que se tengan seriamente en cuenta a aquellas personas cuyos derechos están amenazados (Comisión Interamericana de Derechos Humanos 2019a, 111). Los ejemplos incluyen las agroindustrias, así como la extractiva y la textil, al igual que la prestación de servicios esenciales o las cadenas de suministro en general, entre otras. 
Las consideraciones anteriores relativas al deber de regular dejan espacio para un mayor desarrollo de la dimensión preventiva de la debida diligencia a nivel poblacional, más allá de las circunstancias ya identificadas por la Corte Interamericana. Al seguir desarrollando este enfoque de la debida diligencia, aplicándolo al contexto de las empresas y los derechos humanos, la Comisión Interamericana preparó el escenario para otras empresas. Al respecto, la industria de alimentos y bebidas, por ejemplo, parece estar lista para este abordaje.

\section{b. Obligación de respetar los derechos humanos}

Más allá de la debida diligencia, también se podría argumentar que la cercanía del Estado con la industria de alimentos y bebidas podría implicar una violación de la obligación de respetar los derechos humanos dentro del SIDH.

En Empresas y Derechos Humanos. Estándares Interamericanos, la Comisión Interamericana señaló que la obligación de respetar los derechos humanos implica que los Estados deben abstenerse de participar en conductas relacionadas con actividades corporativas que pongan en peligro los derechos humanos. Por ejemplo, al adoptar acuerdos comerciales y/o de inversión que vayan en contra de las obligaciones de derechos humanos, así como al proveer asistencia o asumir el control sobre empresas, tanto públicas como privadas, que impliquen violaciones de derechos humanos, incluso cuando esto ocurra en relación con organismos internacionales vinculados a actividades corporativas (Comisión Interamericana de Derechos Humanos 2019a, 69). Cabe destacar que cuanto más cerca está una empresa del Estado, y cuanto más depende de una entidad pública o del apoyo del contribuyente, mayor es la necesidad de asegurar el respeto del Estado por los derechos humanos (Comisión Interamericana de Derechos Humanos 2019a, 69).

Posteriormente, la Comisión Interamericana investigó las circunstancias en las que las acciones u omisiones de las empresas 
podrían llevar a que el Estado sea considerado responsable de violar directamente la obligación mencionada. Específicamente, examinó en detalle las siguientes situaciones: empresas que desempeñan funciones propias del poder público (Comisión Interamericana de Derechos Humanos 2019a, 71), que reciben órdenes del Estado o son efectivamente controladas por éste (72), y que realizan un acto que el Estado reclama y adopta unilateralmente como propio (73).

Sin embargo, la Comisión Interamericana dio un paso más allá, indicando que esta lista puede ampliarse bajo la doctrina de la complicidad cuando exista aquiescencia, tolerancia o colaboración del Estado con respecto a los actos en cuestión. Aunque estos factores se han analizado en torno a temas específicos dentro del SIDH, como en el caso de acciones de grupos paramilitares, la Comisión Interamericana aclaró que existe una base jurisprudencial para su continuo desarrollo en el contexto de las empresas y los derechos humanos (Comisión Interamericana de Derechos Humanos 2019a, 74). En este caso, se deben considerar ciertos parámetros, como la cercanía entre el Estado y las empresas y el nivel de patrocinio, coordinación, protección, permisibilidad, tolerancia o inacción mostrado por el Estado en relación con los abusos cometidos por las empresas (75).

A este respecto, es útil establecer un paralelismo con el enfoque de complicidad de los Principios Rectores de las Naciones Unidas. Los Principios Rectores de las Naciones Unidas recurren tanto a definiciones no jurídicas como jurídicas de complicidad, pero las aplican a las empresas en lugar de al Estado. En este sentido, la atención se centra en si una determinada empresa puede ser percibida como cómplice de los actos de otra parte, o puede ser considerada cómplice de la comisión de un delito que conlleve responsabilidad penal o de un daño que conlleve responsabilidad civil (Oficina del Alto Comisionado de las Naciones Unidas, 2012). El contexto en el que operan las empresas puede aumentar el riesgo de que sean consideradas cómplices de graves abusos de los derechos humanos, por lo cual, a la luz de los Principios Rectores de las 
Naciones Unidas, este riesgo es un tema de cumplimiento legal (Oficina del Alto Comisionado de las Naciones Unidas, 2011).

En cambio, la Comisión Interamericana explora el concepto de complicidad en relación con la obligación de respetar los derechos humanos que recae sobre los Estados. Como se ha dicho anteriormente, esto implicaría aquiescencia, tolerancia o colaboración con los actos de terceros, vías que podrían explorarse más a fondo en función de la cercanía entre el Estado y las empresas, teniendo en cuenta el nivel de patrocinio, coordinación, protección, permisibilidad, tolerancia o inacción mostrado por el Estado con respecto a los abusos cometido por las empresas.

En cuanto al tema de la complicidad en relación con las dietas poco saludables, la relación entre los Estados y las empresas puede ser más o menos evidente. En algunos casos, podría adoptar la forma de ayuda explícita a la industria de alimentos y bebidas. En Brasil, por ejemplo, activistas han denunciado que el gobierno está subsidiando la producción de bebidas gaseosas mediante exenciones fiscales al almíbar de azúcar en una región en particular, lo que conlleva beneficios en la compra de dicho producto para las empresas embotelladoras de todo el país (Johns et al. 2020). En otros casos, la complicidad puede ser más sutil, manifestándose a través de la captura corporativa del Estado según lo definido por la Comisión Interamericana: la captura de instituciones públicas o la influencia indebida de las empresas en las y los tomadores de decisiones, para beneficio de las empresas (Comisión Interamericana de Derechos Humanos 2019a, 39).

$\mathrm{Al}$ respecto, es relevante recurrir a otro informe temático de la Comisión Interamericana, titulado Corrupción y Derechos Humanos: Estándares Interamericanos (Comisión Interamericana de Derechos Humanos 2019b). En éste, se analizó el papel de los actores privados en el contexto de la corrupción, abordando el problema de la toma de decisiones que sirve a intereses privados más que al bien común. En particular, la Comisión Interamericana se refirió a dos conceptos: 
“captura del Estado" y “macrocorrupción.” El primero se define como una forma de corrupción en la que los actores privados tienen el poder de influir en la toma de decisiones de las autoridades, logrando beneficios a través de una dinámica que genera dependencia. El segundo es un concepto marco que congrega formas sistémicas de corrupción, esquemas ilícitos que van más allá de las personas y no son necesariamente jerárquicos, que coexisten en una compleja red sin límites territoriales claros, junto con acciones lícitos e ilícitos, al igual que agentes estatales y no estatales (Comisión Interamericana de Derechos Humanos 2019b).

En su forma agravada, la macrocorrupción puede incluso considerarse un concepto independiente. La Comisión Interamericana utilizó como ejemplo la cooptación de instituciones, explicando que "[e]sta forma de corrupción se caracteriza por la captura una institución del Estado a través de actos lícitos e ilícitos, y ésta se pone al servicio de intereses de actores estatales y no estatales, desnaturalizando sus funciones ordinarias" (Comisión Interamericana de Derechos Humanos 2019b, 111). Los ejemplos citados incluyen la penetración y captura de aduanas, de los sistemas de seguridad social y de las obras públicas, que han significado no solo la apropiación indebida de grandes fondos públicos, sino también la imposibilidad de que estas instituciones cumplan con su propósito (Comisión Interamericana de Derechos Humanos 2019b).

Al cooptar procesos de toma de decisiones, incluidos los que conducen a políticas que se aplican a su propia actividad económica, la industria de alimentos y bebidas establece una relación verdaderamente promiscua con el Estado. Las tácticas bien documentadas de esa industria derivan en el deterioro de la capacidad regulatoria de las instituciones pertinentes; un estado de cosas que eventualmente podría enmarcarse como patrocinio, coordinación, protección, permisibilidad, tolerancia o inacción por parte del Estado. En este sentido, es útil acoplar la lectura de Empresas y Derechos Humanos: Estándares Interamericanos con la lectura de Corrupción y Derechos Humanos: Estándares Interamericanos, dado 
que la captura corporativa es clave para entender la obligación de respetar los derechos humanos en relación con la complicidad.

Por último, vale la pena considerar si existe un incumplimiento sostenido por parte del Estado en cuanto a prevenir la violación de los derechos humanos por parte de la industria de alimentos y bebidas. En el Caso 11.227, Funcionarios y miembros de la Unión Patriótica (UP), respecto de Colombia, la Comisión Interamericana argumentó (i) que se habían producido múltiples y sucesivas violaciones del derecho a la vida, desapariciones forzadas y desplazamientos a gran escala; y ii) que hubo una convergencia en el incumplimiento de las obligaciones de proteger y respetar los derechos humanos, "en supuestos de actuación directa, aquiescencia, tolerancia, colaboración y también un incumplimiento flagrante y sostenido del deber de prevención" (Comisión Interamericana de Derechos Humanos 2018).

Si bien este caso tiene sus particularidades, sienta las bases para considerar la obligación de respetar los derechos humanos a la luz de la reiterada falta de prevención de las violaciones de los derechos humanos a través de la aquiescencia, la tolerancia o la colaboración de los Estados. Es, por lo tanto, un concepto útil para el ámbito de las empresas y los derechos humanos, incluida la industria de alimentos y bebidas.

\section{Conclusión}

La amenaza que plantea la industria de alimentos y bebidas para el disfrute de los derechos humanos no es un daño colateral. Como dice Freudenberg, "los ejecutivos de las empresas de alimentos siempre están en busca de un producto de gran éxito, uno que se venda solo, que gane nuevos consumidores y produzca ganancias generosas" (Fredeunberg 2014, 7). Parte de esta búsqueda radica en comercializar y publicitar agresivamente productos no saludables, mientras que, al mismo tiempo, las empresas burlan las regulaciones 
que limitarían su capacidad para hacerlo, como se analiza a lo largo de este artículo. Los Estados también tienen la culpa de esta situación. Capturados por la industria de alimentos y bebidas, han permitido que la formulación de políticas públicas sirva a los intereses de estas empresas a expensas de los derechos humanos.

En este artículo analizamos esta situación con base en la obligación de garantizar los derechos humanos, en relación con la debida diligencia, y con base en la obligación de respetar los derechos humanos, en relación con el concepto de complicidad, a la luz de dos recientes informes temáticos emitidos por la Comisión Interamericana, así como la jurisprudencia de la Corte Interamericana. Aunque aún queda mucho por hacer, nuestra conclusión es que, dentro del SIDH, los Estados pueden ser considerados responsables por su incumplimiento de la obligación de garantizar los derechos humanos; en particular, por no actuar con la debida diligencia mediante la regulación efectiva de la industria de alimentos y bebidas. Además, más allá de la debida diligencia, argumentamos que los Estados también pueden ser considerados responsables de incumplir la obligación de respetar los derechos humanos. En primer lugar, porque la estrecha relación entre el Estado y la industria de alimentos y bebidas puede enmarcarse como patrocinio, coordinación, protección, permisibilidad, tolerancia o inacción del primero con respecto a la segunda. En segundo lugar, debido al flagrante y sostenido incumplimiento del Estado de la obligación de prevenir violaciones de los derechos humanos por parte de la industria de alimentos y bebidas.

Los Estados no pueden esperar que la industria de alimentos y bebidas resuelva un problema que no solo creó, sino que también siegue alimentando, incluso en medio de la pandemia de la Covid-19. Después de todo, mientras no hay evidencia alguna de la efectividad de la autorregulación, sí hay pruebas de sobra respecto a lo que sucede cuando no se controla la industria de alimentos y bebidas. Por lo tanto, es necesario aplicar los marcos de derechos humanos existentes, entre ellos en el SIDH, al contexto de las dietas poco 
saludables. Más que confiar en que las empresas van a respetar los derechos humanos, los Estados deben actuar; o de lo contrario, ser considerados responsables por su propia falla en garantizar y/o respetar los derechos humanos.

\section{Referencias bibliográficas}

Abramovich, Víctor. (2010). "Responsabilidad estatal por violencia de género: comentarios sobre el caso "Campo Algodonero" en la Corte Interamericana de Derechos Humanos." Anuario de Derechos Humanos 6: 167. 10.5354/0718-2279.2011.11491.

Anand Grover (Special Rapporteur on the right of everyone to the enjoyment of the highest attainable standard of physical and mental health). Rep. on Unhealthy foods, non-communicable diseases and the right to health. U.N. Doc. A/HRC/26/31 (April 1, 2014)

Cabrera, Oscar, y Andrés Constantin. "Tobacco control in the InterAmerican Human Rights System." In Human Rights and Tobacco Control. Edward Elgar Publishing, 2020.

Caso Velásquez Rodríguez Vs. Honduras. Fondo. Corte Interamericana de Derechos Humanos (Julio 29 de 1988).

Caso Ximenes Lopes Vs. Brasil. Fondo. Corte Interamericana de Derechos Humanos (Julio 4 de 2006).

Caso González y otras (“Campo Algodonero”) Vs. México. Excepción Preliminar, Fondo, Reparaciones y Costas, Corte Interamericana de Derechos Humanos (Noviembre 16 de 2009).

Caso Suárez Peralta Vs. Ecuador. Excepciones Preliminares, Fondo, Reparaciones y Costas, Corte Interamericana de Derechos Humanos (Mayo 21de 2013).

Caso Gonzales Lluy y otros Vs. Ecuador. Excepciones Preliminares, Fondo, Reparaciones y Costas, Corte Interamericana de Derechos Humanos (Septiembre 1 de 2015).

Caso Trabajadores de la Hacienda Brasil Verde Vs. Brasil. Excepciones Preliminares, Fondo, Reparaciones y Costas, Corte Interamericana de Derechos Humanos (Octubre 20 de 2016) 
Caso Poblete Vilches y otros Vs. Chile. Fondo, Reparaciones y Costas. Sentencia de 8 de marzo de 2018., Corte Interamericana de Derechos Humanos (Marzo 8 de 2018).

Caso Cuscul Pivaral y otros Vs. Guatemala. Interpretación de la Sentencia de Excepción Preliminar, Fondo, Reparaciones y Costas, Corte Interamericana de Derechos Humanos (Agosto 23 de 2018).

Caso Hernández Vs. Argentina. Excepción Preliminar, Fondo, Reparaciones y Costas, Corte Interamericana de Derechos Humanos (Noviembre 2 de 2019).

Caso Comunidades Indígenas Miembros de la Asociación Lhaka Honhat (Nuestra Tierra) Vs. Argentina, Corte Interamericana de Derechos Humanos (Febrero 6 de 2020)

Caso Empleados de la Fábrica de Fuegos en San Antônio de Jesus y sus familiares vs. Brasil, (Excepciones Preliminares, Fondo, Reparaciones y Costas), Corte Interamericana de Derechos Humanos (Julio 15 de 2020)

Colectivo de Abogados José Alvear Restrepo y El Poder del Consumidor. 2020. "La interferencia de la industria es nociva para la salud. Estrategias corporativas contra el etiquetado frontal de advertencia: un estudio comparado de Chile, Perú, México y Uruguay." https://colectivodeabogados.org/interferenciaetiquetado/imag enes/Interferencia industria etiquetado latam 25sept.pdf

Comisión Interamericana de Derechos Humanos. Julio 25 de 2018. "CIDH presenta caso sobre Colombia ante la Corte IDH" https://www.oas.org/es/cidh/prensa/Comunicados/2018/162.a sp

Comisión Interamericana de Derechos Humanos. "Empresas y Derechos Humanos: Estándares Interamericanos" OEA/Ser.L/V/II. CIDH/REDESCA/INF.1/19. (1 de noviembre de 2019).

Comisión Interamericana de Derechos Humanos. "Corrupción y Derechos humanos: Estándares Interamericanos" OEA/Ser.L/V/II. Doc. 236. (Diciembre 6 de 2019).

Comité de Derechos Económicos, Sociales y Culturales. "Observaciones finales sobre el cuarto informe periódico 
de la Argentina" Documento ONU E/C.12/ARG/CO/4 (1 de noviembre de 2018).

Comité de Derechos Económicos, Sociales y Culturales. "Observaciones finales sobre el quinto informe periódico de Mauricio" Documento ONU E/C.12/MUS/CO/5 (5 de abril de 2019).

FAO, PAHO, WFP y UNICEF. 2019. "Panorama de la seguridad alimentaria y nutricional en América Latina y el Caribe 2019." https://iris.paho.org/bitstream/handle/10665.2/51685/9789251 319581FAO_spa.pdf? sequence $=6$.

Freudenberg, Nicholas. 2014. Lethal But Legal: Corporations, Consumption, and Protecting Public Health. New York: Oxford University Press.

Global Food Research Program at the University of North Carolina at Chapel Hill. July 2020 (updated). "Fact Sheet 'Industry SelfRegulation: Empty Pledges'." https://globalfoodresearchprogram.web.unc.edu/files/2020/08 /Industry-Self-regulation-\%E2\%80\%93-Empty-pledges\%E2\%80\%93-July-2020.pdf

Gostin, Lawrence. 2014. Global Health Law. Cambridge, Massachusetts; London, England: Harvard University Press.

Gostin, Lawrence. 2016. “'Big Food' is Making America Sick'” Milbank Q. Sept; 94(3): 480-484.

Grupo REFORMA. March 17, 2020. "Piden posponer etiquetado ante COVID-19".

https://testigos.intermediaweb.com.mx/testigos/clip_reporte_ histo_ssl.php? clave $=50339688$.

Hilal Elver (Special Rapporteur on the Right to Food). Interim report of the Special Rapporteur on the right to food. U.N. Doc A/71/282 (August 3, 2016)

Jean Ziegler (Special Rapporteur on the right to food). Rep. on The right to food. U.N. Doc. A/56/210 (July 23, 2001)

Johns, Paula. Adriana Ramos, Marques Casara. September 13, 2020. "A destruição da Amazônia e o subsídio aos refrigerantes." NEXO. 
https://www.nexojornal.com.br/ensaio/2020/A-

destrui\%C3\%A7\%C3\%A3o-da-Amaz\%C3\%B4nia-e-osubs\%C3\%ADdio-aos-refrigerantes

Kickbusch, Ilona, Luke Allen, Christian Franz. 2016. "The Commercial Determinants of Health". The Lancet Global Health 4,2: e895896. https:// doi.org/10.1016/S2214-109X(16)30217-0.

Lee, Kelley and Eric Crosbie. July, 2020. “Understanding Structure and Agency as Commercial Determinants of Health. Comment on 'How Neoliberalism is Shaping the Supply of Unhealthy Commodities and What This Means for NCD Prevention." International Journal of Health Policy Management 9, 7: 315-318. https://doi.org/10.15171/ijhpm.2019.127

Mialon, Melissa. 2020. "An overview of the commercial determinants of health." Globalization and Health 16, no. 1:1-7. https://doi.org/10.1186/s12992-020-00607-X

Moodie, Rob, David Stuckler, Carlos Monteiro, Nick Sheron, Bruce Neal, Thaksaphon Thamarangsi, Paul Lincoln, Sally Caswell, on behalf of The Lancet NCD Action Group. 2013. "Profits and pandemics: prevention of harmful effects of tobacco, alcohol, and ultra-processed food and drink industries." The Lancet 381 : 670-79. http://dx.doi.org/10.1016/S0140-6736(12)62089-3.

NCD Alliance, SPECTRUM Consortium. September 9, 2020. "Signalling Virtue, Promoting Harm - Unhealthy commodity industries and COVID-19". https://ncdalliance.org/resources/signalling-virtue-promotingharm.

Nestle, Marion. 2015. Soda Politics: Taking on Big Soda (and Winning). New York: Oxford University Press.

Oficina del Alto Comisionado de las Naciones Unidas para los Derechos Humanos. 2012. "La responsabilidad de las empresas de respetar los derechos humanos. Guía para la interpretación”. https://www.ohchr.org/Documents/Publications/FAQ_Principl esBussinessHR_SP.pdf

Oficina del Alto Comisionado de las Naciones Unidas para los Derechos Humanos. July 27, 2020. "Statement by the UN Special Rapporteur on the right to health on the adoption of front-ofpackage warning labelling to tackle NCDs." 
https://www.ohchr.org/EN/NewsEvents/Pages/DisplayNews.aspx?

NewsID=26130\&LangID=E\#: :text=The\%20right\%20to\%20healt h\%20and,of\%20physical\%20and\%20mental\%20health

Una traducción no oficial al español se encuentra en: https://www.dejusticia.org/wpcontent/uploads/2020/10/Traduccion-etiquetado-frontalONU-1.pdf

Organización Panamericana de la Salud. 2011. "Recomendaciones de la Consulta de Expertos de la Organización Panamericana de la Salud sobre la promoción y publicidad de alimentos y bebidas no alcohólicas dirigida a los niños en la Región de las Américas.”

Organización Panamericana de la Salud. 2019.“Enfermedades no transmisibles: hechos y cifras" https://iris.paho.org/handle/10665.2/51482

Organización Panamericana de la Salud. 2019. “Alimentos y bebidas ultraprocesados en América Latina: ventas, fuentes, perfiles de nutrientes e https://iris.paho.org/handle/10665.2/51523 implicaciones"

Organización Panamericana de la Salud. Agosto 28 de 2020. "Respuesta al brote de COVID-19 en la Región de las Américas." https://www.paho.org/es/documentos/respuesta-al-brotecovid-19-region-americas

Organización Panamericana de la Salud. Septiembre 28 de 2020. "El etiquetado frontal como instrumento de política para prevenir enfermedades no transmisibles en la Región de las Américas" https://iris.paho.org/bitstream/handle/10665.2/52740/PAHON MHRF200033 eng.pdf? sequence $=6 \&$ isAllowed $=\mathrm{y}$

Organización Panamericana de la Salud. Septiembre 29 de 2020. El etiquetado frontal como instrumento de política para prevenir enfermedades no transmisibles en la Región de las Américas". https://www.paho.org/es/documentos/etiquetado-frontalcomo-instrumento-politica-para-prevenir-enfermedades-no-0

Organización Panamericana de la Salud. Dieciembre 26 de 2020. "Enfermedades no transmisibles". https://www.paho.org/es/temas/enfermedades-notransmisibles 
Organización Mundial de la Salud. 2013.“Global action plan for the prevention and control of noncommunicable diseases 2013$2020 "$.

https://apps.who.int/iris/bitstream/handle/10665/94384/97892 41506236_eng.pdf? sequence $=1$.

Organización Mundial de la Salud. Convenio Marco para el Control del Tabaco, Febrero 27de 2005. https://apps.who.int/iris/bitstream/handle/10665/42813/92435 91010.pdf?sequence $=1$

Organización Mundial de la Salud. Junio 1 de 2018. "Enfermedades no transmisibles. Datos y cifras." https://www.who.int/es/newsroom/fact-sheets/detail/noncommunicable-diseases

Popkin, Barry M. Sufa Du, William D. Green et al. 2020. "Individuals with obesity and COVID-19: A global perspective on the epidemiology and biological relationships." Obesity Reviews 21, no. 11: 1-17. https://doi.org/10.1111/obr.13128.

Rivera, Humberto Fernando Cantú. 2017. "Debida diligencia en derechos humanos: Breves reflexiones." Derechos humanos y empresas: reflexiones desde América Latina. Instituto Interamericano de Derechos Humanos.

Roache, Sarah A. Charles Platkin, Lawrence Gostin, Cara Kaplan. 2018. "Big Food and Soda Versus Public Health: Industry Litigation Against Local Government Regulations to Promote Healthy Diets.” The Fordham Urban Law Journal 45(4), 1051-1089.

Tangcharoensathien, V, Chandrasiri, O., Kunpeuk, W., Markchang, K., Pangkariya, N. 2019. "Addressing NCDs: Challenges From Industry Market Promotion and Interferences" International journal of health policy and management 8, 5: 256-260. https://doi.org/10.15171/ijhpm.2019.02.

The Lancet Global Health. (2019) "Stop industry interference, save lives." The Lancet Global Health 7, no. 12: e1584. 10.1016/S2214109X(19)30469-3

Unaids.org. Agosto 27 de 2020. "Las lecciones aprendidas de la respuesta al VIH: ONUSIDA advierte de todos los peligros que conlleva el no respetar los derechos humanos en la respuesta a la COVID-19."

ONUSIDA https://www.unaids.org/es/resources/presscentre/pressrelease 
andstatementarchive/2020/august/20200827_rights-in-apandemic

United Nations Interagency Task Force on NCDs. 2020. "Policy Brief 'Covid-19 and NCD risk factors.'

https://www.who.int/docs/default-source/ncds/uninteragency-task-force-on-ncds/uniatf-policy-brief-ncds-andcovid-030920-poster.pdf?ua $=1$ 